\title{
Prevalence and antibiotics resistance pattern of extended spectrum $\beta$ - lactamases Escherichia coli strains isolated from chickens
}

\author{
Ali Zaman ${ }^{1}$, Shakeeb Ullah ${ }^{1}$, Syeda Nayab Batool Rizvi ${ }^{2}$, Kamran \\ Safdar $^{1}$, Khalid Muhammad ${ }^{1}$, Asif Javaid ${ }^{1}$, Umer Khitab ${ }^{3}$, \\ Muhammad Inam Malik ${ }^{1}$, Niamat Ullah ${ }^{1}$ and Muhammad Shuaib \\ $\mathrm{Khan}^{1 *}$ \\ 1. Faculty of Veterinary and Animal Sciences, Gomal University, D.I. Khan-Pakistan \\ 2. Institute of Chemistry, University of Punjab-Pakistan \\ 3. Islamic international University Islamabad-Pakistan \\ *Corresponding author's email: shoaibbaloch2002@yahoo.com \\ Citation \\ Ali Zaman, Shakeeb Ullah, Syeda Nayab Batool Rizvi, Kamran Safdar, Khalid Muhammad, Umer Khitab, \\ Muhammad Inam Malik, Niamat Ullah and Muhammad Shuaib Khan. Prevalence and antibiotics resistance \\ pattern of extended spectrum $\beta$-lactamases Escherichia coli strains isolated from chickens. Pure and Applied \\ Biology. Vol. 7, Issue 1, pp207-220. http://dx.doi.org/10.19045/bspab.2018.70025
}

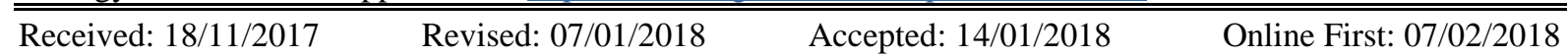

\section{Abstract}

Current work was planned to demonstrate the antimicrobial resistance pattern and prevalence of extended spectrum $\beta$-lactamase (ESBL)-producing Escherichia coli and its subtypes from chicken. Antimicrobial resistance of $E$. coli strains was conducted by Disc Diffusion while ESBL strains were detected by Double Disc Synergy methods. The lower prevalence of the ESBLs strains from the blood samples of healthy chickens (12\%) as compared to intestinal contents (13\%). The most prevalent ESBLs strains was determined the inducible cephalosporinases bush functional gene $2 \mathrm{e}(5-20 \%)$ followed by carbapenamases (4-10\%). The least resistance of E.coli isolates was observed against quinolone group $(30-56 \%)$ from blood samples of healthy broilers as compared to cephalosporin group (78-86\%)while intermediate resistance was noted against metallo $\beta$-lactam (imipenem and carbapenems; $48 \%$ ). Higher resistance was recorded against amoxiclave (68\%). Minimum resistance of the E.coli isolates showed against ciprofloxacin (30\%) while against cepharadine (100\%). Higher resistance was observed against cephalosporin from $78-100 \%$. Furthermore, the $E$. coli isolates from birds resisted to metallo $\beta$-lactam antimicrobials (imipenem, 45\% and carbapenems, 47\%). In general, the lowest resistance (40\%) was recorded against ciprofloxacin and ofloxacine but 89 and 100\% resistance was observed against cephtrioxone and cepharadine, respectively. Furthermore, the quinolone group of the antimicrobial agents was noted as least active against the isolates. Higher prevalence of ESBLs isolates was determined from the feces $(51 \%)$ on the ground followed by the intestinal contents of diseased chickens (49\%). It was concluded that ESBLs strains of E. coli in bird found highly resistant to majority of antibiotics against them.

Keywords: Birds; Cephalosporinases; Carbapenamases; Disc diffusion; ESBLs strains

\section{Introduction}

In many countries of the world, numbers of antimicrobial preparations are used without any instruction. Latest study on the Disease
Control and Prevention (CDC) recognized the excessive usage of broad-spectrum antimicrobial drugs by office-based physicians in the United States for the treatment of 
sinusitis otitis medium and other common infections [1]. Resistance of various types is express by Gram-negative bacteria. Cooperation of and multidrug efflux pumps and barrier of outer membrane resulted in the intrinsic resistance. Extra modern antibacterial like broad-pectrum $\beta$-lactams and fluoroquinolones are selected mostly for overproduction of mutants from the pumps and resulted in bacterial resistant to mostly antibacterial classes' agents [2]. Another eneral process in Gram-negative bacteria is the enzymes' production that is capable to destroy antimicrobial preparations. Latest types of such enzymes are in progress to defeat the "last chance antibiotics" like the azteonam, cefepime and ceftazidime which are known as extended spectrum $\beta$-Lactamases (ESBL), evolved from the SHV and TEM $\beta$-lactamases and are plasmid borne mostly found in E. coli, Klebsiella pneumoniae, and other genera of Enterobacteriaceae [3]. Escherichia coli strains are commonly harmless, few serotypes may resulted in humans and poultry' infections [4]. The flora of the gut normally contain noninfectious strains that by produce vitamin $\mathrm{K}$ [5] and prevent the development of pathogenic bacteria $[4,5]$. Gut flora contain about $0.1 \%$ of E. coli and related bacteria. Bacterial pathogenic strains transmitted mainly from fecal oral transmission [6]. In birds, E. coli is ordinarily existed and disease may activated through several events, leading to disease syndromes range like as infection of respiratory tract, yolk sac infection, omphalitis, cellulitis, septicaemia and swollen head syndrome $[7,8]$. The enzymes $\beta$-lactamases are classified on the basis of the amino acid and nucleotide sequences. Up till now according to functional classification there are four (A-D) recognized classes. A, C and D class acts via the mechanism based on serine, but for the action of metallo- $\beta$-lactamases or B class zinc is required. The commonly ESBLs are related to A class [9], defined with an approximately 29000 Da molecular mass, the penicillins' preferential hydrolysis and the serine activesite. [10]. The ESBL of TEM-type is derived from TEM 1 and 2. In France 1984, Klebsiella pneumoniae samples were observed, named
CTX-1 due to enhanced action for cefotaxime were plasmid-mediated B-lactamases [11]. This enzyme currently known as TEM-3 differs by two amino acid substitutes from TEM-2 [12]. More than 100 TEM-type ßlactamases are described, out of which mostly are ESBLs. Some TEM ß-lactamases' mutants were being isolated. They preserve the capability of hydrolyzing 3rd-generation cephalosporins and also act as an inhibitor resistance. CTX-M family currently defined from the Extended-Spectrum B-Lactamases group [13]. CTX name reveals the potency of hydrolysis activity for cefotaxime [14]. The said enzymes can hydrolyze well to cephalothin than benzyl penicillin and favourably hydrolyze cefotaxime more than the ceftazidime. Ceftazidime MICs are generally in superficial prone range, few CTXM- type Blactamases consult resistance [15]. Aztreonam MICs have been found to be flexible. Blactamases of CTX-M-type hydrolyzes efficiently to the cefepime [16]. The PER-type has a share of about $25-27 \%$ with the known SHV and TEM- types of ESBLs [17]. The PER-1 B-lactamase, first time isolated from the Pseudomonas aeruginosa's strains in Turkey [18], later on isolated from Salmonella entericase, Acinetobacter baumanii, Alcaligenes fecalis, Porteus mirabilis and Typhimurium, [16]. PER-1 B-lactamase which is susceptible to clavulanic acid inhibition hydrolyzes efficiently cephalosporins and penicillin. VEB-1 is most homologous (38\%) to PER-1 and 2. It consults more resistance's level to ceftazidime, aztreonam and cefotaxime, inversed by clavulanic acid. VEB1 , first time detected in Vietnam, in the single E.coli' isolate [19]. In Thailand, an identical beta-lactamase, found in Pseudomonas aeruginosa, K. pneumonia and Enterobacter cloaca isolates [20]. In China and Kuwait other VEB enzymes have been also discovered [15]. The resistant bacteria may disturb the food chain, resulting in food safety problems. Earlier research performed in Portugal explained the distribution of $E$. coli isolates containing ESBL from the faecal microbiota of wild animals, dogs, and especially in prey birds and recently in swine and chickens $[21,22, \&$ 
23]. This study was conducted with the aims to investigate the Extended Spectrum $\beta$ Lactamases E.coli strains' prevalence in chicken and to estimate the antibiotic resistance of different strains of $E$ coli samples isolated from chickens against antibiotics.

\section{Materials and methods}

In this study 250 samples were collected from broilers, representing all age groups selected at random including both normal and diseased. Out of total, 100 blood samples were taken in vacutainer tubes from both, diseased (50) and normal (50) broilers. Furthermore, 100 samples of intestinal contents of both, diseased (50) and normal (50) chickens and also 50 samples of faecal material on the ground were taken in sterile polythene bags from poultry farm (s) in Tandojam Pakistan. The samples were brought and processed at Veterinary Microbiology Laboratory, Department of Veterinary Microbiology, Sindh Agriculture University (SAU), Tandojam. The Fecal, blood and intestinal content samples from poultry farms were collected and processed according to standard procedure and then cultured on the differential media (initially MacConkey Agar). The culture media was incubated for 24 hours at $35-37{ }^{\circ} \mathrm{C}$. The suspected $E$. coli colonies (pink, mucoid, positive string test) were picked by sterilized wire loop and immediately cultured on a fresh MacConkey agar plate by streaking plate method until pure culture was obtained. Gram's staining was carried out by a standard protocol.

\section{Process}

The microbes were recognized by using API 20E up to level of species (15 samples), Standard biochemical tests [24]. The samples used on selective differential media for E. coli, MacConkey inositol penicillin agar) and Eosine Methylene blue agar (EMB Agar). The E. coli's resistance design was determined for selected battery of antibiotics [25]. The minimum inhibitory concentration (MIC) on minimum bactericidal concentration (MBC) was done as procedure adopted by National
Committee for Clinical laboratory Standards [26]. Inhibitor Resistant Tem $\beta$ Lactamases gene detection, carbapenamase production, bush functional genes, Extended Spectrum $\beta$-Lactamases and inducible chromosomal $\beta$-lactamase were determined by using antibiotic susceptibility testing by CDS (Calibrated Dichotomous Susceptibility Tests, 2004).

Storage and maintaining of isolated samples

The isolated samples were streaked with the slants of tryptic soya agar and then preserved at $4^{\circ} \mathrm{C}$.

\section{Biochemical test for identification}

Following standard biochemical tests were carried out for identification of microbes up to species level:

SIM agar test (hydrogen sulphide, indole and motility test)

The microorganisms' pure culture was introduced by puncture into the butt. A sterilized straight wire-loop was used for this purpose and the inoculated tubes were incubated an aerobically, at $35{ }^{\circ} \mathrm{C}$ for 18 $24 \mathrm{hrs}$. The growth was observed only along with line showing immobility. The $\mathrm{H}_{2} \mathrm{~S}$ formation with black colour in tube was recorded as positive to indole test, performed by adding KOVAC's indole reagent [27].

\section{TSIA (Triple Sugar Iron Agar Test)}

Pure culture was lined by a central stab on the sloped surface and butt of the same tube. The inoculated tube was then incubated for 48 hours at $37^{\circ} \mathrm{C}$.

\section{Simmon citrate test}

The pure culture was streaked on the surface of the culture medium and incubated at $35{ }^{\circ} \mathrm{C}$ for 24 - $48 \mathrm{hrs}$.

\section{Methyl red and VOGES-PROSKAUER}

Two tubes containing MR-VP broth were inoculated at $35{ }^{\circ} \mathrm{C}$ up to $24 \mathrm{hrs}$ with the pure culture of bacterial organism and incubated. After incubation, carried out methyl red test. Furthermore, a test VOGES-PROSKAUER was also carried out by adding $1 \mathrm{ml}(40 \%)$ of potassium hydroxide solution and $3 \mathrm{ml}$ BARRIT's solution. 


\section{Urease test}

The medium was inoculated massively by the pure culture under investigation and incubated for 5-48 hours at $35^{\circ} \mathrm{C}$ [28].

\section{Lysine decarboxylase}

The medium was incubated at $35^{\circ} \mathrm{C}$ for 24 hours after the inoculation with the organisms.

\section{Oxidase test}

Oxidase reagent $(1 \%)$ was freshly prepared by dissolving $0.5 \mathrm{~g}$ tetra-methyl-para phenylene-diamine di-hydrochloric acid in $5 \mathrm{ml}$ distilled water. A sterilized filter strip was impregnated with 2-3 drops of this reagent and a colony of test strain was smeared on this filter paper. The red color appeared is considered as positive.

\section{Differential media}

\section{MacConkey agar MacConkey}

A pure culture was streaked on the culture medium's surface a

\section{Eosine methylene blue (EMB) (Fluka Biochemical)}

After sterility confirmed, the isolated Escherichia coli, colony was streaked on the surface than incubation was done for 24 hours at $37^{\circ} \mathrm{C}$ and observed for colony morphology.

\section{Sensitivity of antibiotic testing}

The sensitivity of antibiotic pattern of Escherichia coli was calculated with the help of Kerby Bauer's Disc diffusion method [25]. The antibiotic discs used are shown in antibiotic listof the The clinicall Lab standard institute [29] criteria were adopted to interpret the diameters of the zones.

\section{Disc diffusion method}

The disc diffusion method was done for each bacterial isolate on Muller Hinton agar (CM337-Oxoid) as the growth medium. About $25 \mathrm{ml}$ of the medium were dispensed in $90 \mathrm{~mm}$, and $60-70 \mathrm{ml}$ of the media in $150 \mathrm{~mm}$ sterilized Petri plates and incubation was done overnight to confirm sterility of medium at $37^{\circ} \mathrm{C}$.

\section{Inoculum preparation}

Tryptic soya broth medium of $5 \mathrm{ml}$ (CM129-Oxoid) was prepared in accordance with the instruction of manufacturer and then suspended in screw caped test tubes for sterilization with the help of autoclave for 15 minute at $121^{\circ} \mathrm{C}$. Then for the medium's sterility cooled and preserved at $35^{\circ} \mathrm{C}$ for 24 hours in an incubator. On next day, single isolate of every recognized clinical sample was put in the sterilized test tube for inoculation at $35^{\circ} \mathrm{C}$ in the incubator for overnight. After 24 hours, turbidity was changed to 0.5 Macfar land standards with the addition of sterile saline.

The sterilized cotton swab was used to spread the inoculum over the surface of Muller Hinton agar plate. Before the applying of discs, plates were dried. With the help of forceps discs of given potencies were applied on the inoculated plates within 15 minutes and placed in inverted position for $18-24$ hours in the incubator at $35^{\circ} \mathrm{C}$. After that, the examination of plates was done and measured the inhibitions' zones in millimetre $(\mathrm{mm})$ with a ruler.

\section{Determination of ESBLs}

The disc synergy method/disc synergy was used to detect the extended spectrum $\beta$ lactamases (ESBL). A disc of co-

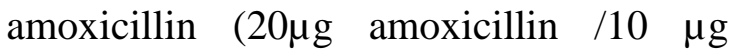
clavulinic acid) was placed in the centre of the agar surface. The discs of cefotaxime, ceftriaxone, ceftazidime and azteronam were arranged in such a way that the distance between the central disk and the surrounding disks were approximately $30 \mathrm{~mm}$. The isolates were incubated at $37^{\circ} \mathrm{C}$ for 24 hours. After an overnight incubation the zones around $3^{\text {rd }}$ generation cephalosporins and azteronam disc were observed. The zones of inhibition were observed next day if there was no extension of the $3^{\text {rd }}$ generation of cephalosporine and azteronam towards co-amoxiclave disc was observed and the organism was considered to be non ESBL producer [30].

\section{Inducible cephalosporinase detection}

Production of chromosomal $\beta$-lactamases is efficiently induced by the Imipenem. Inducible chromosomal $\beta$-lactamases were detected by using CDS method (Calibrated Dichotomous Susceptibility Tests, 2004). 
Cephalosporin disc of $20 \mathrm{~mm}$ was kept adjacent to $10 \mu \mathrm{g}$ Imipenem disc, it resulted in boosting of progress of both cephalosporin and the imipenem discs and cephalosporin inhibitory zone's edges were flattened.

\section{Detection of inducible cephalosporinases of bush functional gene $2 \mathrm{e}$}

The inducible cephalosporinases were detected by CDS method. The inducible cephalosporinases were recognized by the flattened edge of inhibitory zone around a cefotaxime $5 \mu \mathrm{g}$ disc.

\section{Detection of inhibitor resistant tem $\beta$ - lactamases (IRTs)}

IRT producing strains are susceptible to cephalexin but resistant to augmentin. The sensitivity pattern of augmentin and cephalexin was noted by using the CDS Method (Calibrated Dichotomous Susceptibility Tests, 2004). Strain sensitive to cephalexine but resistant to augmentin were regarded as TEM $\beta$-lactamases producing strain

\section{Detection of carbapenamase producing strains}

These are zinc-dependent metallo-enzymes can hydrolyse the ertapenem, imipenem, meropenem, carbapenems and the penicillin. The carapenamase production was determined by using CDS method. In this case the inhibitory zone around the imipenum / meropenum was noted and resistant strains were documented as carbapenamase producing strain.

\section{Results}

\section{The isolation and identification of Extended Spectrum $\beta$-lactamases $E$. coli from broiler chicken}

A total of 250 samples, 50 each from blood of normal and diseased, intestinal contents of all age groups and further 50 only from faecal material of the chickens present on the ground of broiler chicken farms were collected and processed. The samples were cultured on specific culture media for isolation and characterization of Escherichia coli. Initial isolation was made on culture media, specifically designed for Escherichia coli and then processed for further confirmation using biochemical test. After isolation and confirmation of Escherichia coli isolates (250), the antibiotic resistance of each isolate was tested against antibiotics to confirm the $\beta$ lactamases Escherichia coli isolate.

The resistance pattern of $E$. coli isolates to antibiotics recovered from blood of healthy broiler chickens

The results regarding resistance pattern of all $E$. coli isolates, isolated from blood of healthy broiler chickens were determined against 15 antibiotics using Disc Diffusion Method are presented in (Figure1). The antibiotics used were: cephalosporins (cephradine, cephalexin 1st, cefeclor 2nd, ceftrioxone, cefotxime 3rd) carbapenems (imipenem, meropenem), quinolones (nalidixic acid), flouroquinolones (ofloxacine, norfloxacine, moxifloxacin, ciprofloxacin, gatifloxacin) amoxicillin/ clavulanic acid and kanamycin. Nearly all strains were found resistant to majority of antimicrobial agents used during the investigation with the exception of nalidixic acid (NA) that was found intermediately effective (40\%) against the isolates. Furthermore, the isolates did resist to other antimicrobial agents that ranged from 4$18 \%$.

The resistance pattern of $E$. coli strains against antimicrobial agents isolated from blood of diseased chicken

The results regarding resistance pattern of E. coli strains obtained from blood samples of diseased broiler chickens during study are given in (Figure 2). The isolates were found highly resistant to majority of antimicrobial agents used against them. The isolates were observed highly susceptibility to cepharadine, cefeclor, cephtrixone, and cephalexine and their susceptibility was recorded as $86,82,80$ and $78 \%$ respectively. The results regarding other antibiotics recorded during investigation are presented in (Figure 2). However, the quinolones group antimicrobial agents were found least effective (30-56\%) against the isolates. The metallo $\beta$ - lactam antimicrobials (imipenem, $48 \%$ and carbapenems, 48\%) 
were also observed as intermediate effective against $E$.coli isolates.

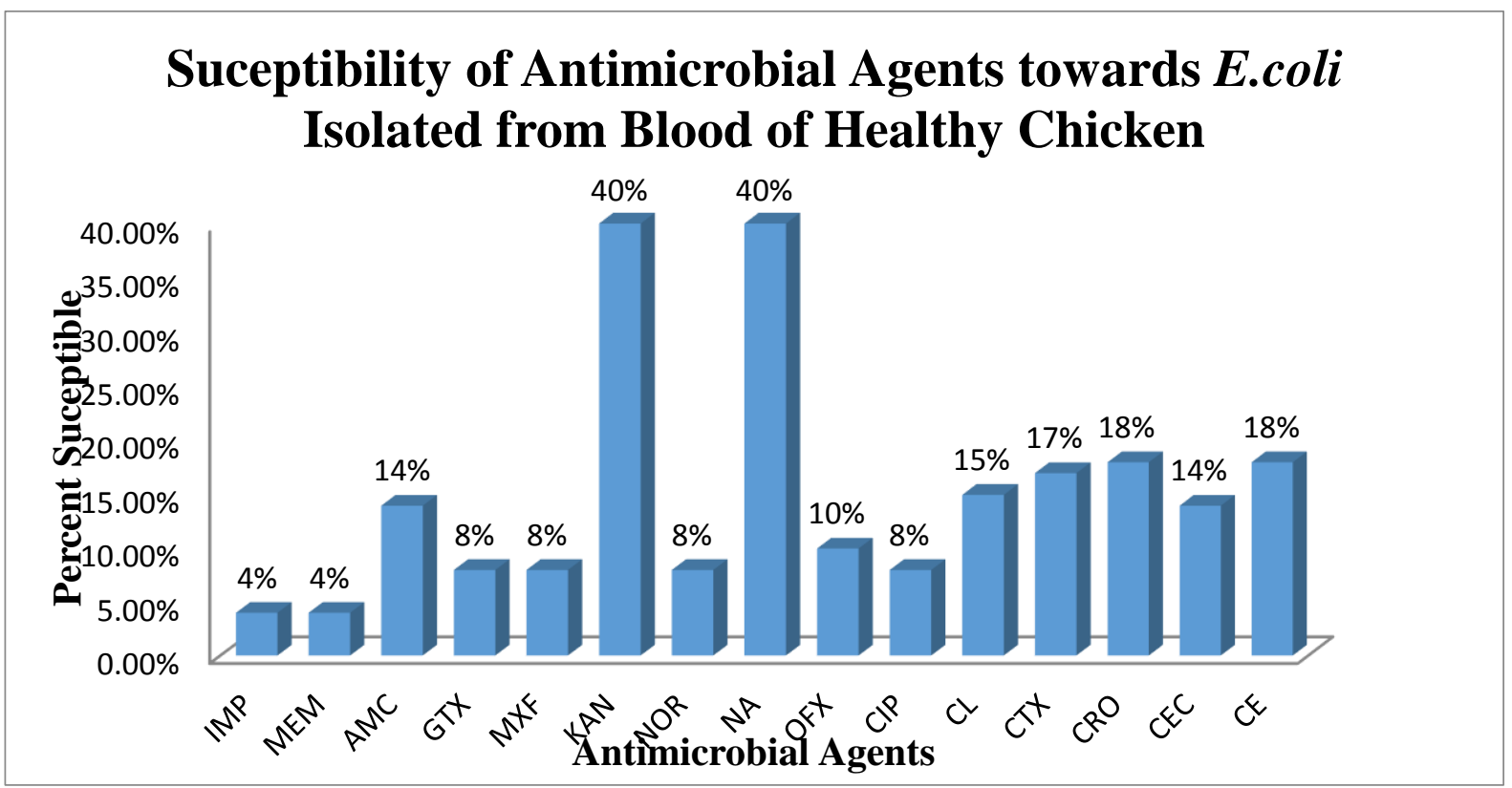

Figure 1. The resistance pattern of antimicrobial agents against $E$. coli isolates, isolated from blood samples of healthy chicken

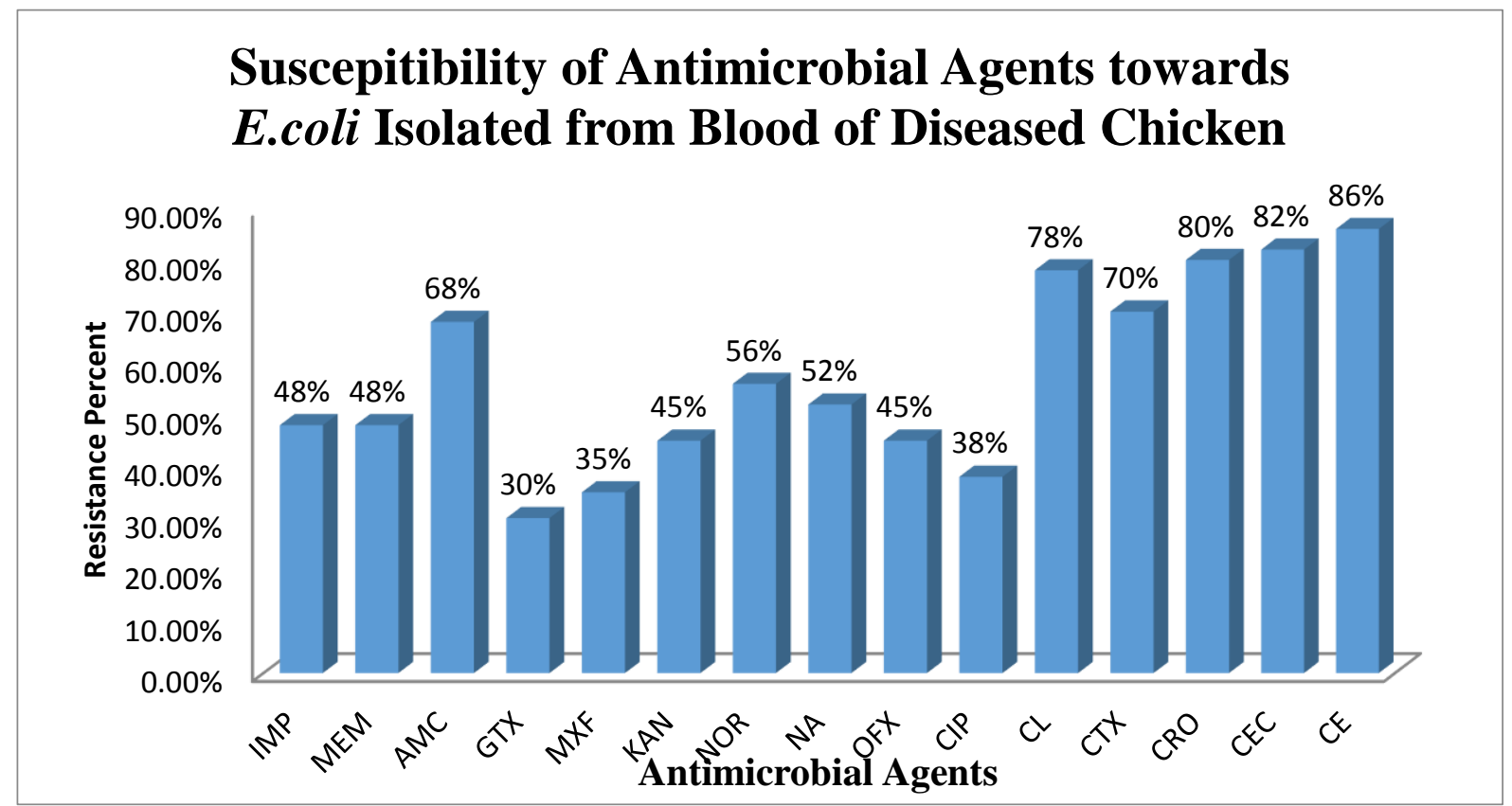

Figure 2.The resistance pattern of antimicrobial agents against $E$. coli isolates obtained from blood of diseased chicken

The resistance pattern of $E$. coli isolates against antibiotics recovered from the intestinal contents of healthy broiler chickens

During current study the resistance pattern of the E. coli isolates obtained from intestinal contents of healthy broiler chickens was also investigated to compare the susceptibility and resistance to antimicrobial agents in between the isolates obtained from blood and intestines of broilers. All the strains isolated from 
intestines of healthy boilers were found least susceptible to majority of antimicrobial agents. The agents were recorded least effective against the $E$. coli strains and their action against the isolates was recorded that ranged from $3-37 \%$. The ciprofloxacin was emerged as highly ineffective antimicrobial agents against isolates and recorded as $3 \%$ whereas the kanamycin was found as $45 \%$ effective drug against the $E$. coli isolated detected from the intestinal contests of healthy broilers. The activity of other drugs against E.coli isolates is also given in the same (Figure 3). Furthermore, it is very interesting that the E. coli isolates obtained from blood of the healthy broilers showed susceptibility to a majority of the antibiotics while resistance to a few antimicrobial agents but here a total different result were recorded in the case of intestinal isolates who did resist to almost all antimicrobial agents. Second is that only few antibiotics showed action against the isolates up to $45 \%$ while others below $37 \%$. One can make a conclusion from the study that the bacterial organisms in the intestines are more resistant as compared to the blood organisms the possible reason might be that the organisms are more experienced to different drugs and chemicals; therefore they develop resistance against the antibiotics as observed in the present survey.

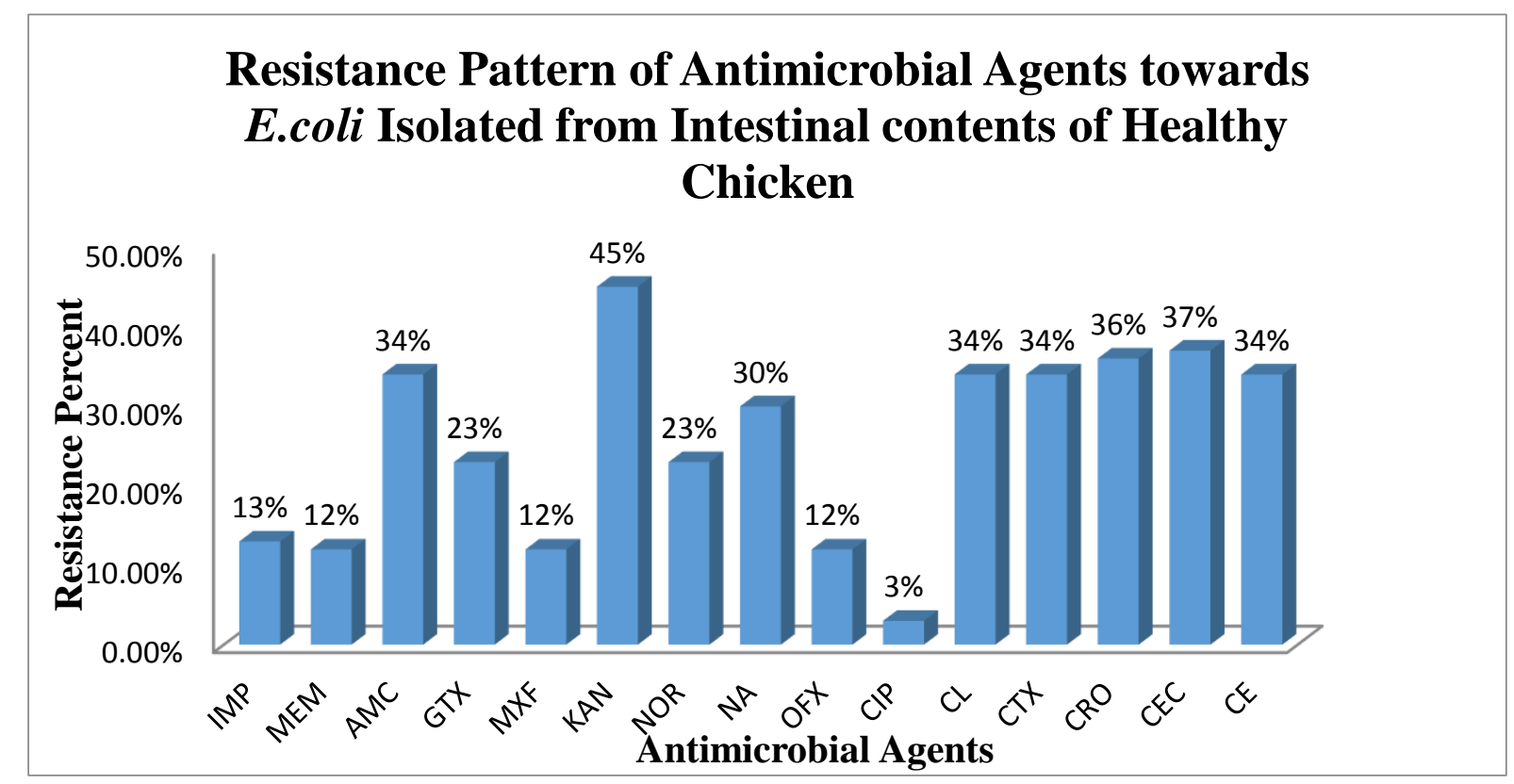

Figure 3.The resistance pattern of antimicrobial agents against $E$. coli isolates, isolated from Intestinal contents of healthy chicken

The resistance pattern of $E$. coli isolates against antimicrobial agents detected from intestinal contents of diseased broiler chickens

Similar investigation was carried out on the resistance pattern of $E$. coli strains recovered from the intestine and blood samples of the diseased and normal broilers against different antibiotics. The results regarding resistance pattern of the E. coli isolates against antibiograms are presented in Figure 4. The isolates of intestinal contents of diseased broiler chickens were found highly resistant to few drugs that ranged from $78-100 \%$ while minimum resistance level was recorded as $30 \%$ against ciprofloxacin whereas $100 \%$ resistance of E.coli isolates showed against cepharadine. On the whole, the strains of the intestinal contents were showed least resistance against quinolone group that ranged from $30-49 \%$ as compared to 
cephalosporin group that ranged from 78$100 \%$. The results of other drugs are given in the same Figure 4. Similar pattern of the resistance was observed in between the isolates obtained from blood and intestine samples of disused broilers. The isolates from both types of samples did resists to the same antibiotics but only difference was observed in the percentage of resistance. Otherwise no big difference was observed during investigation in both types of isolates of $E$. coli recovered from blood and intestinal samples of diseased broilers.
However, a clear difference between the isolates obtained from healthy broilers and diseased broiler chickens. The isolates obtained from diseased broilers were found highly resistant to majority of the antibiotics used against them as compared to healthy isolates. The reason might be that they are in active position and secreting more resistant enzymes (Extended spectrum $\beta$-lactamases) could inhibit the activity of the antibiotics and showed highly resistant to antibiotics.

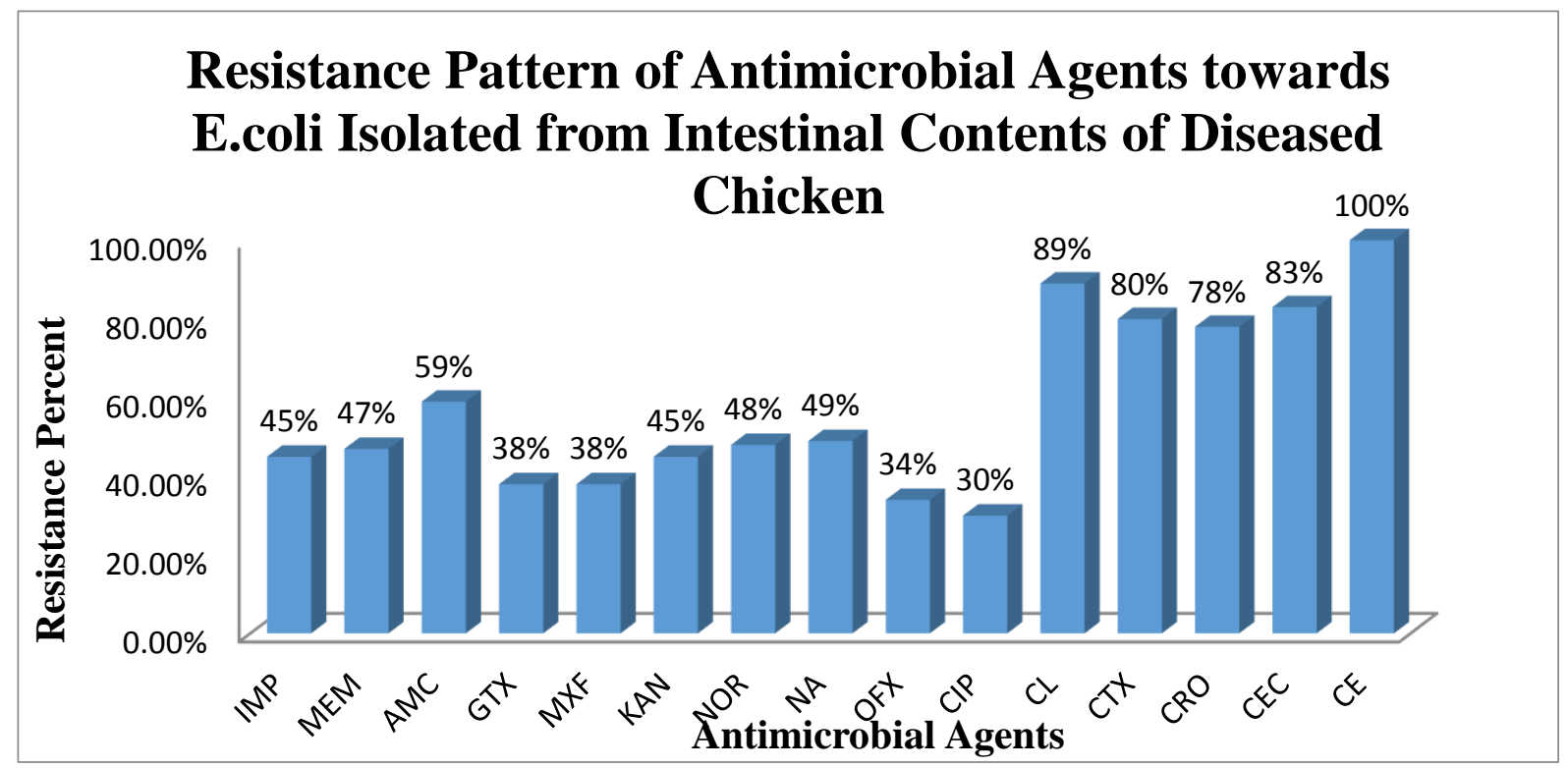

Figure 4. The resistance pattern of antimicrobial agents against $E$. coli isolates, isolated from intestinal contents of diseased chicken

The resistance pattern of $E$. coli against antibiotics isolates, isolated from the feces of boiler chickens

The resistance pattern of $E$. coli strains isolated from faeces of chicken seen throughout the investigation was found extremely resistant; they showed their range from $42-100 \%$. It was recorded that resistance observed in case of E.coli isolated from faeces was alarmingly elevated compared to that of blood and intestinal contents. In general lowest resistance levels were observed as $40 \%$ in case of ciprofloxacin and ofloxacine whereas cephtrioxone and cepharadine presented 89 and $100 \%$ resistance respectively towards
E.coli. On the whole the quinolone group of the antimicrobial agents was observed as least effective (40-49\%) as compared to cephalosporin group (67-100\%). The metallo beta lactam antimicrobials (imipenem and carbapenem) presented intermediate levels of resistance (46 and $47 \%$ respectively). The amoxiclave /clavulanic acid also presented high resistance levels $(60 \%)$

The prevalence of ESBLs isolates recovered from blood, intestinal contents and feces of broiler chickens

During investigation the E. coli isolates, obtained from various sources were processed for the presence of ESBLs and its 
subtypes. The higher prevalence of ESBLs isolates was recorded from the faeces of broilers $(51 \%)$, followed by the intestinal contents of diseased chicken $(49 \%)$ while $45 \%$ prevalence of the ESBLs strains was recorded from the blood samples of diseased broiler chickens. A lower prevalence $(12 \%)$ of ESBLs isolates was recoded from blood of healthy chickens as compared to the intestinal contents of the healthy chicken (13\%). It is clear from the study that higher prevalence of ESBLs was recorded in faeces of the broilers while second higher prevalence was observed in the blood samples of diseased broilers. The reason for higher prevalence of ESBLs in faecal material might be due to the role of environment that contributed and changed ( mutation) the normal isolates in to ESBLs where all birds either diseased and normal excretes the E.coli in their faeces that are on the ground the environment developed in them the nature of ESBLS. Otherwise there is no solid reason to record and discuss for its/ their role in developing the ESBLs nature or property in common E.coli. But in the case of blood, the reason is clear that blood of birds provide all nutritional requirements to the E.coli strains, second is that the pathogenic bacteria are active in nature could develop such property of ESBLs.

\section{The prevalence of ESBLs subtypes in boilers recognized by double disc synergy method}

During present study, the ESBLs isolates recognized from various sample sources were further processed to identify the strains through in-vitro detection of the ESBLs subtypes. In case of $E$. coli isolated from blood of the healthy chicken the most prevalent subtype was detected as inducible cephalosporinase bush functional gene $2 \mathrm{e}$ (5\%), followed by carbapenamase $(4 \%)$. While in case of $E$. coli isolated from blood of the diseased chickens, the most prevalent ESBLs subtype IRT was (25\%) followed by carbapenamase (10\%). Likewise the carbapenamases (9\%) was observed the most prevalent ESBLs subtype of $E$. coli isolated from the intestinal contents of the healthy chicken. The higher prevalence (20\%) of inducible cephalosporinase bush functional gene $2 \mathrm{e}$ was seen in the strains isolated from faeces of the chicken.

\section{Discussion}

Antibiotics are widely used in poultry production as growth promoters or to control infectious disease. The unnecessary use of antibiotics caused high resistance to antimicrobial agents in normal chicken flora $[31,32]$ and pathogenic organisms [33].

In the current resistance pattern of the $E$. coli isolated from blood of healthy chicken was determined and results revealed a lower resistance levels. In facts, all strains were susceptible to majority of antimicrobial agent used during the investigation and the prevalence of resistance was significantly lower as reported previously by many workers [34]. However, the E.coli isolated from the blood of diseased chicken showed higher resistance levels as reported previously $[35,36]$. In the present study majority of the E.coli isolates did resist to cephalosporins (all generation). Most probable reason for this finding is the presence of extended spectrum $\beta$-lactamses E.coli that generally hydrolyzed the $\beta$ lactam ring of the cephalosporin antimicrobials resulting in the prevalence of resistance [37].

Conversely comparably lower resistance levels were determined in carbapenem group of the antimicrobial agents as demonstrated previously [38]. Most probable cause may be the zwitteronic structure of the carbapenems that protects the drugs from hydrolysis [18]. The quinolones showed better results during investigation. It presented better efficacy against E. coli isolated from blood of diseased chicken [27, 39].

Intestinal microflora is generally regarded as most important source for the spread of antibiotic resistance thus resulting in spread of serious ailments [7]. In this regard, the E.coli strains were isolated from intestinal contents of both, healthy and diseased chicken to screen out for resistance pattern. 
It was noted that susceptibility ratio of the strains obtained from healthy chicken intestinal contents was better as compared to diseased chicken despite the fact that the healthy chicken possessed resistant strains [40]. In the same context the cephalosporins were recorded as equally resistant to E.coli isolates and inhibit the growth of the organism obtained from the both, the intestinal contents of healthy and diseased chicken, however, the higher prevalence of E.coli was recoded in diseased chicken. The findings regarding the prevalence of $E$. coli demonstrated in this observation are in agreement to the findings recorded by other workers [41].

The carbapenems are generally found less active against the Gram-negative microbes on account of limited use and zwitteronic structure that protects the $\beta$-lactam ring to hydrolyze $[42,43]$. Surprisingly less effective and higher resistance levels were noted of $E$. coli isolates against carbapenems recovered from both healthy and diseased chickens intestinal contents. However, less resistance levels were observed against the drugs, the E. coli strains isolated from the healthy chicken as compared to diseased ones. Whereas it was observed that, carbapenems presented less action against E.coli as compared to all other antibiotic groups. The resistance of $E$. coli against carbapenems was considered to be the presence of carbapenamases genes that are responsible to deactivate the carbapenems [44]. The findings of the present survey regarding resistance of E.coli isolates to carbapenems are in line to the other workers [45]. They also recorded similar pattern of resistance as observed in this study.

The amoxicillin/clavulanic acid is a combination of antimicrobial agent and is being used in the feed for a variety of reasons [39]. During present investigation an increased resistance was seen against amoxicillin/clavulanic acid by E.coli isolated from both healthy and diseased chicken whereas less resistance was in this investigation observed as compared to the workers reported previously [34]. The higher resistance levels against a combination antimicrobial agent are an indicator of spread of antibiotic resistance [39]. Likewise during the present investigation, the quinolone antimicrobials were found the most effective drugs against E.coli isolated from intestinal contents of both diseased and healthy chicken. The ciprofloxacin was determined as highly effective antimicrobial agent. The results recorded in this survey about antibiotics are in agreement to the other workers [36] who also mentioned the similar findings in their reports regarding the antibiotics against E.coli isolated from different sources.

The results of the present investigation revealed extremely elevated levels of the resistance to nearly all antimicrobial agents against the $E$. coli isolated from obtained feces. The resistance range for the E. coli isolated from the faeces was alarming irrespective of the spectrum of activity. Exact explanation of this is the presence of ESBLs that facilitate hydrolysis of the antimicrobial agent leading to spread of antimicrobial resistance [44]. Despite the metallo-beta lactam ring of carbapenems, the resistance level seen in these antimicrobial agents was elevated has been reported previously [44]. Likewise the amoxicillin /clavulanic acid presented high resistance levels that was recorded as another evidence for the presence of ESBLs [46, 47]. Nevertheless, quinolone antimicrobials presented comparably better susceptibility towards the E.coli isolated from chicken feces. Limited use of these antimicrobial agents in the poultry was regarded as the most suitable explanation of these results.

The higher resistance of E.coli strains was observed against cephalosporins antibiotics and amoxicillin/clavulanic acid was an indication of ESBLs property in these strains. The higher prevalence of the ESBLs strains was recorded in faeces followed by intestinal contents of diseased chicken during present study. These finding are in accordance with the $[26,46]$. Furthermore, 
the ESBLs positive isolates were further screened out to confirm the various ESBLs subtypes. It was evident that the Inducible cephalosporinase bush functional gene $2 \mathrm{e}$ is prevailing in all E. coli isolates detected from various sources during present investigation.

Investigations were undertaken to demonstrate the prevalence and antimicrobial resistance of extended spectrum $\beta$ lactamases and its subtypes among $E$. coli isolated from chicken. Antimicrobial susceptibility was performed by Disc Diffusion Method while ESBLs and its subclass were detected by Double Disc Synergy method. The least resistance (30$56 \%$ ) of E.coli isolates recovered from blood specimen of healthy and diseased chicken was recorded against quinolone group of antimicrobial agents as compared to cephalosporin group that ranged from 78$86 \%$. While intermediate resistance was noted against metallo $\beta$-lactam (imipenem and carbapenems) and measured as $48 \%$. However, somewhat higher resistance as (68\%) was recorded against amoxiclave /clavulanic acid. Generally, the E. coli isolates obtained from intestinal contents of both, healthy and diseased chicken showed higher resistance against majority of the antibiotics up to $100 \%$. The minimum resistance of the E.coli isolates showed against ciprofloxacin and was recorded as $30 \%$, whereas against cepharadine it was determined as $100 \%$. On the whole the $E$. coli isolates were found least resistant (30$49 \%$ ) to quinolone group of the antimicrobial agents while highly to cephalosporin group that ranged from 78$100 \%$. Further that the E. coli isolates detected from birds did resist to metallo $\beta$ lactam antimicrobials (imipenem, $45 \%$ and carbapenems, 47\%). Likewise the samples from faeces of the chicken were recorded as most heavily polluted with resistant E.coli. In general, the lowest resistance $(40 \%)$ was recorded against ciprofloxacin and ofloxacine whereas 89 and $100 \%$ resistance was observed against cephtrioxone and cepharadine respectively. Furthermore, the quinolone group of the antimicrobial agents was noted less active against the isolates. The higher prevalence of ESBLS isolates was determined from the faeces $(51 \%)$ on the ground followed by the intestinal contents of diseased chickens (49\%).

The lower prevalence of the ESBLs strains was observed from the blood samples of healthy chicken (12\%) as compared to intestinal contents of the healthy chickens (13\%). The most prevalent ESBLs subtypes was determined the inducible cephalosporinases bush functional gene $2 \mathrm{e}$ $(5-20 \%)$ followed by carbapenamases (4$10 \%)$.

\section{Conclusions}

E. coli isolates recovered from the blood and intestinal contents of diseased boilers are more resistant as compared to the isolates obtained from the blood and intestinal contents of healthy broilers, showed $100 \%$ resistance against cepharadine.

The E.coli strains isolated from the faeces of the broilers showed similar pattern of resistance to the majority of the antibiotics as recorded for the isolates detected from the intestinal and blood samples of diseased broilers during present study.

An investigation was also carried out to recognize the ESBLs from the samples obtained from blood, intestinal contents and feces of healthy and diseased broilers were tested through In-vitro double disc synergy method. The most prevalent subtypes were detected as inducible cephalosporinases bush functional gene $2 \mathrm{e}(5 \%)$, followed by carbapenamase $(4 \%)$ while from blood of the diseased chickens, the most prevalent ESBLs subtype IRT (25\%) was recognized followed by carbapenamase (10\%). Similarly, the carbapenamases $(9 \%)$ was observed, the most prevalent ESBLs subtype of $E$. coli from the intestinal contents of the healthy chicken. Whereas the higher prevalence $(20 \%)$ of inducible cephalosporinases bush functional gene $2 \mathrm{e}$ strain was isolated from the feces of the chickens. 


\section{Authors' contributions}

Conceived and designed the experiments: A Zaman, S Ullah, A Javaid \& MS Khan, Performed the experiments/ Review materials: A Zaman, U Khitab, MI Malik \& N Ullah, Analyzed the data/ Editing: S Ullah, A Javaid \& MS Khan, Contributed reagents/ materials/ analysis tools: A Zaman, S Ullah \& A Javaid, Wrote the paper: SNB Rizvi, K Safdar, K Muhammad \& MS Khan.

\section{References}

1. McCaig LF \& Huges JM (1995). Trends in antimicrobial drug prescribing among office -based physicians in United States. JAMA 273: 214-219.

2. Li Z \& Nikaido H (2004). Effluxmediated drug resistance in bacteria. Drugs 64: 159-204.

3. Samaha-kfoury J N \& Araj GF (2003). Recent developments in $\beta$ lactamases and extended spectrum $\mathrm{B}$-lactamases. $\mathrm{Br} \mathrm{Med}$ J 327: 1209-1213.

4. Vogt RL \& Dippold L (2005). Escherichia coli O157:H7 outbreak associated with consumption of ground beef, June-July, 2002. Pub Heal Rep 120: 174-78.

5. Bentley R \& Meganathan R (1982). Biosynthesis of vitamin $\mathrm{K}$ (menaquinone) in bacteria. Microbiol Rev 46(3): 241-80.

6. Hudault S, Guignot $\mathrm{J} \&$ Servin AL (2001). Escherichia coli strains colonizing the gastrointestinal tract protect germ-free mice against Salmonella typhimurium infection. Gut 49(1): 47-55.

7. Morley AJ \& Thomson DK (1984). Swollen-head syndrome in broiler chickens. Avian Dis 28: 238-243.

8. Randall C J, Meakins PA, Harris MP \& Watt DJ (1984). A new skin disease in broilers. Vet Rec 114: 246.

9. Ambler RP (1980). The structure of $\beta$ lactamases, Philos Trans $R$ Soc London B 289: 321-331.

10. Medeiros A, Mayer KH \& Opal SM (1988). $\quad$ Plasmid-mediated $\quad \beta$ - lactamases, Antimicrob. News Letter 5: 61-65.

11. Burn-Buisson C, Legrand P, Philippon A, Montravers F, Asquer M \& Duval J (1987). Transferable enzymatic resistance to third generation cephalosporins during nosocomial outbreak of multi resistant Kelbsiella pneumoniae. Lancet 11: 302-306.

12. Sougakoff W, Goussard S, Gerbaud G \& Courvalin P (1988). Plasmidmediated resistance to third generation cephalosporins caused by point mutations in TEM-type penicillinase genes. Rev Infect Dis 10: 879-884.

13. Tzouvelekis L S, Tzelepi E, Tassios PT \& Legakis NJ (2000). CTX - M - type beta-lactamases: an emerging group of extended spectrum enzymes. Int $J$ Antimicrob Agents 14: 137-142.

14. Bonnet R (2004). Growing group of extended-spectrum $\beta$-lactamases: the CTX-M enzymes. Antimicrobl Chemoth 48: 1-14.

15. Poirel L, Rotimi VO, Mokaddas EM, Karim A \& Nordmann P (2001). VEB1 like extended - spectrum betalactamases in Psuedomonas aeruginosa, Kuwait. Emerg Infect Dis 7: 468-470.

16. Vahaboglu H, Ozturk R \& Aygun G (1997). Wide spread detection of PER1-type extended-spectrum B-lactamases among nosocomial Acinetobacter and Psuedomonas aerurginosa isolates in Turkey: a nationwide multicentre study. Antimicrob Chemoth 41: 2265-2269.

17. Nordmann P \& Nass T (1994). Sequence analysis of PER-1 extended spectrum $\quad \beta$-lactamase from Psuedomonas aeruginosa and comparison with class A $\beta$-lactamases. Antimicrob Chemoth 38: 104-114.

18. Nordmann P, Ronco E, Nass T, Duport C, Michel-Briand Y \& Labia R (1993). Characterization of a novel extendedspectrum beta-lactamase from Psuedomonas aeruginosa. Antimicrob Chemoth 37: 962-969. 
19. Poirl L, Nass T, Guibert M, Chaibi B, Labia R \& Nordmann P (1999). Molecular and biochemical characterization of VEB-1, a novel class A extended-spectrum $\beta$-lactamase encoded by an Escherichia coli integron gene. Antimicrob Chemoth 43: 573581.

20. Girlich D, Poirel L, Leelaporn A, Karim A, Tribuddharat C, Fennewald M \& Nordmann P (2001). Molecular epdimiology of the integron-located VEB-1 extended - spectrum betalactamase in nosocomial enterobacterial isolates in Bangkok, Thailand. J Clin Microbiol 39: 175-182.

21. Costa D, Poeta P, Briñas L, Sáenz Y, Rodrígues J \& Torres C (2004). Detection of CTX-M-1 and TEM-52 $\beta$ Lactamases in Escherichia coli strains from healthy pets in Portugal. $J$ Antimicrobl Chemoth 54: 960-961.

22. Costa D, Laura V, Patricia P, Ana CC, Manuela M, Yolanda S, Sergio S, Myriam Z, Jorge $\mathrm{R}$ \& Carmen, $\mathrm{T}$ (2009). Prevalence of extendedspectrum beta-lactamase-producing Escherichia coli isolates in faecal samples of broilers. Vet Microbiol 138: 339-44.

23. Machado E, Coque TM, Canton R, Sousa JC \& Peixe L (2008). Antibiotic resistance integrons and extendedspectrum $\quad \beta$-lactamases among Enterobacteriaceae isolates recovered from chickens and swine in Portugal. $J$ Antimicrobial Chemoth 62: 296-302.

24. Bergeys DH (1992). Manual of Determinative Bacteriology, William and Wilkins Co Baltimore $7^{\text {th }} \mathrm{Ed}, \mathrm{Pp}$ : 230-231.

25. Bauer A, Kirby W, Sherris JC \& Turck M (1966). Antibiotic susceptibility testing by a standardized single disk method. Am J Clin Pathol 45: 493-496.

26. Dierikx C, Van Essen-Zandbergen A, Veldman K, Smith H \& Mevius D (2010). Increased detection of extended spectrum beta-lactamase producing Salmonella enterica and Escherichia coli isolates from poultry. Vet Microbiol 145: 273-38.

27. Sulkin SE \& Willet JC (1940). A TSI ferrous sulphate medium for use identification of enteri organisms. $J$ Lab Cli Med 25: 649-653.

28. Rustigian R \& Stuart CA (1941). Decomposition of urea by proteus. Pro Soc Expl Mol 4: 178-182.

29. National Committee for Clinical Laboratory Standards (1997) Performance standards for antimicrobial disk susceptibility tests. Approved standard M2-A6. (National Committee for Clinical Laboratory Standards, Wayne, $\mathrm{Pa}$ ).

30. Jarlier V, Nicola MH, Fournier G \& Philippon A (1988). Extended broadspectrum $\beta$-lactamases conferring transferable resistance to newer $\beta$ lactam agents in Enterobacteriaceae: hospital prevalence and susceptibility patterns. Rev Infect Dis 10: 867-878.

31. Allan BJ, Hurk VD \& Potter JV (1993). Characterization of Escherichia coli isolated from cases of avian colibacillosis. Can J Vet Res 57(3): 146-151.

32. Linton A H (1977). Antibiotics, animals and man. An appraisal of a continuous subject. In: Antibiotics and Antibiosis in Agriculture (M Woobine), Butterworth, London, Pp: 315-343

33. Amara A, Ziani Z \& Bouzoubaa K (1995). Antimicrobial resistance of Escherichia coli strains isolated in Morocco from chickens with colibacillosis. Vet Microbiol 43: 325330.

34. Miles TD, Lwayne $\mathrm{N} \&$ Paul DB (2006). Antimicrobial resistance of E.coli isolation from broiler, chicken and human BMC. Vet Res 2:7.

35. Hassan MR, Alam KD, Mahjabeen S \& Rehman M F (2011). Free Redical Sca Pharmacalogy online 2: 774-703.

36. Wang H \& Liu YM (2003). Mechanism of carbapenems resistance in Acinetobacter baumannii. Zhongguo Yi 
Xue Ke Xue Yuan Xue Bao 25(5): 56772.

37. Livermore DM (2008). Defining an extended-spectrum beta-lactamase. Clin Microbiol Infect 14: 3-10.

38. Islam M J, Sultana S, Das KK, Sharmin N \& Hassan MN (2008). Isolation of plasmid mediated multidrug resistant $E$. coli from poultry. Int J Sustain Crop Prod 3(5): 46-50.

39. Al-Ghamdi MS, El-Morsy F, AlMustafa ZH, Al-Ramadhan M \& M. Hanif (1999). Antibiotic resistance of Escherichia coli isolated from poultry workers, patients and chicken in the eastern province, Saudi Arabia. Trop Med Int Health 4: 278-83.

40. Ono K, Kaneko H, Choudhary S \& Pilbeam CC (2005). Biphasic effect of e2 on E.coli dugs. J bone Min Res 20: 23-29.

41. Bergeron AR, Catharine P, Boerlin P \& Daignault D (2011). Chicken as reservoir for extra-intestinal pathogenic Escherichia coli in humans. Can Emer Inf Dis 18: 3.13.

42. Jacoby GA (1997). Extended-spectrum $\beta$-lactamases and other enzymes providing resistance to oxyimino-betalactams. Infect Dis Clin N Am 11: 875887.

43. Poirel L, Gniadkowski M \& Nordmann $P$ (2002). Biochemical analysis of the ceftazidime-hydrolysing extended- spectrum beta-lactamase CTX-M-15 and of its structurally related betalactamase CTX-M-3. J Antimicrob Chemoth 50: 1031-1034.

44. Rossolini GM, D'Andrea MM \& Mugnaioli C (2008). The spread of CTX-M-type extended-spectrum betalactamases. Clin Microbiol Infect 14: 33-41.

45. Lavilla S, Tortola MT, Miro E, Gonzalez JJ, Larrosa N, Sabate M, Navarro F \& Prats G (2005). First detection of a carbapenem-hydrolyzing metallo enzyme in two Enterobacteriaceae isolates in Spain. Antimicrob Chemoth 49: 3492-3494.

46. Overdevest I, Willemsen I, Rijnsburger M, Eustace A, Xu L, Hawkey P, Heck M, Savelkoul P, Vandenbroucke-Grauls $\mathrm{P}$, Zwaluw $\mathrm{K}$, Huijsdens $\mathrm{X}$ \& Kluytmans J (2011). ExtendedSpectrum $\beta$-Lactamase. Genes of Escherichia coli in chicken meat and humans. The Nether Emerg Infect Dis $17: 12$

47. Smet A, Martel A, Persoons D, Dewulf J, Heyndrickx M, Catry B, Herman L, Haesebrouck F \& Butaye P (2008). Diversity of extended-spectrum blactamases and class $\mathrm{C}$ b-lactamases among cloacal Escherichia coli isolates in Belgian broiler farms. Antimicrob Chemoth 52: 1238-43. 